\title{
Influence of ambiguity precision on the success rate of GNSS integer ambiguity bootstrapping
}

\author{
P. J. G. Teunissen
}

Received: 12 March 2006 / Accepted: 14 October 2006 / Published online: 10 November 2006

(C) Springer-Verlag 2006

\begin{abstract}
In this contribution, we study the dependence of the bootstrapped success rate on the precision of the GNSS carrier phase ambiguities. Integer bootstrapping is, because of its ease of computation, a popular method for resolving the integer ambiguities. The method is however known to be suboptimal, because it only takes part of the information from the ambiguity variance matrix into account. This raises the question in what way the bootstrapped success rate is sensitive to changes in precision of the ambiguities. We consider two different cases. (1) The effect of improving the ambiguity precision, and (2) the effect of using an approximate ambiguity variance matrix. As a by-product, we also prove that integer bootstrapping is optimal within the restricted class of sequential integer estimators.
\end{abstract}

Keywords GNSS ambiguity resolution · Integer bootstrapping $\cdot$ Ambiguity precision

\section{Introduction}

Global Navigation Satellite System (GNSS) ambiguity resolution is the process of resolving the unknown cycle ambiguities of double difference (DD) carrier phase data as integers. Ambiguity resolution applies to a great variety of GNSS models that are currently in use in navigation, surveying, geodesy and geophysics. An overview of these models, together with their applications, can be found in textbooks such as Hofmann-Wellenhof et al.

P. J. G. Teunissen ( $\varangle)$

Delft Institute for Earth Observation and Space Systems

(DEOS), Delft University of Technology, Kluyverweg 1,

Delft 2629 HS, The Netherlands

e-mail: P.J.G.Teunissen@tudelft.nl
(1997), Leick (1995), Misra and Enge (2001), Parkinson and Spilker (1996), Strang and Borre (1997), and Teunissen and Kleusberg (1998).

Any GNSS model can be cast in the following system of linear(ized) observation equations

$y=A a+B b+e$

where $y$ is the given GNSS data vector of order $m ; a$ and $b$ are the unknown parameter vectors, respectively, of order $n$ and $p$; and where $e$ is the noise vector. Matrices $A$ and $B$ are assumed known. The data vector $y$ will usually consist of the 'observed minus computed' single-, dual- or triple-frequency DD carrier phase and/or pseudorange (code) observations accumulated over all observation epochs. The entries of vector $a$ are then the DD carrier phase ambiguities, expressed in units of cycles rather than range. They are known to be integers, $a \in Z^{n}$. The entries of the vector $b$ will consist of the remaining unknown parameters, such as for instance baseline components (coordinates) and possibly atmospheric delay parameters (troposphere, ionosphere). They are known to be real-valued, $b \in R^{p}$.

The procedure which is usually followed for solving the linear GNSS model (1), can be divided into three steps. In the first step, one simply discards the integer constraints $a \in Z^{n}$ on the ambiguities and performs a standard least-squares adjustment. As a result, one obtains the (real-valued) estimates of $a$ and $b$, together with their variance-covariance matrix

$\left[\begin{array}{l}\hat{a} \\ \hat{b}\end{array}\right],\left[\begin{array}{cc}Q_{\hat{a}} & Q_{\hat{a} \hat{b}} \\ Q_{\hat{b} \hat{a}} & Q_{\hat{b}}\end{array}\right]$

This solution is referred to as the 'float' solution. In the second step, the 'float' ambiguity estimate $\hat{a}$ is used to 
compute the corresponding integer ambiguity estimate

$\check{a}_{S}=S(\hat{a})$

with $S: R^{n} \mapsto Z^{n}$ a mapping from the $n$-dimensional space of real numbers to the $n$-dimensional space of integers. Once the integer ambiguities are computed, they are used in the third and final step to correct the 'float' estimate of $b$. As a result one obtains the ambiguity resolved baseline solution

$\check{b}_{S}=\hat{b}-Q_{\hat{b} \hat{a}} Q_{\hat{a}}^{-1}\left(\hat{a}-\check{a}_{S}\right)$

This solution is usually referred to as the 'ambiguity fixed' baseline. The quality of the estimator $\breve{b}_{S}$ depends on the quality of the 'float' solution, $\hat{a}$ and $\hat{b}$, and on the quality of the integer estimator $\breve{a}_{S}$. Different choices of the map $S: R^{n} \mapsto Z^{n}$, will result in different integer estimators and will thus also produce differences in the probability distribution of the 'fixed' baseline (Teunissen 1999a).

In this contribution, we concentrate on the second step and consider the principle of integer bootstrapping. In particular, we study the dependence of its probability of correct integer estimation on the variance matrix of the ambiguity float solution. For that purpose, we first give a brief review of the theory of integer bootstrapping in Sect.2. We also show in this section that the bootstrapped estimator is a member from the class of sequential integer estimators. This result enables us later to determine an, albeit restricted, optimality property of integer bootstrapping, somewhat similar to the optimality of integer least-squares as proven in Teunissen (1999b).

In Sect. 3, we study the effect of the ambiguity precision on the probability of correct integer estimation from bootstrapping. Although the bootstrapped estimator is very easy to compute, it does not take all the information of the ambiguity variance matrix into account. This raises the question whether it takes sufficient information into account to profit from any possible precision improvement of the ambiguities. We prove that this is the case, i.e. that the probability of correct integer estimation of bootstrapping will always get larger when the precision of the ambiguity float solution improves.

In Sect. 4, we study what happens to the performance of bootstrapping if an improper ambiguity variance matrix is used. It is shown that, with one exception, the probability of correct integer estimation always gets smaller if either a too optimistic or a too pessimistic precision description is used (in the one exceptional case, the probability remains the same).

\section{Integer bootstrapping}

\subsection{The bootstrapped estimator}

Integer bootstrapping is based on the principle of sequential conditional least-squares estimation. In order to describe the process of integer bootstrapping, we start from the principle of conditional least-squares estimation. We have the following result from standard adjustment theory (Teunissen 2000).

Conditional least-squares Let the expectation and dispersion of $\hat{a}_{I}=\left(\hat{a}_{1}, \ldots, \hat{a}_{i-1}\right)^{T} \in R^{i-1}$ and $\hat{a}_{i} \in R$ be given as

$E\left\{\left[\begin{array}{l}\hat{a}_{I} \\ \hat{a}_{i}\end{array}\right]\right\}=\left[\begin{array}{l}a_{I} \\ a_{i}\end{array}\right], \quad D\left\{\left[\begin{array}{c}\hat{a}_{I} \\ \hat{a}_{i}\end{array}\right]\right\}=\left[\begin{array}{cc}Q_{I} & Q_{I i} \\ Q_{i I} & \sigma_{i}^{2}\end{array}\right]$

Then the least-squares estimator of $a_{i}$, when $a_{I}$ is constrained to the fixed vector $z_{I}$, is given as

$\hat{a}_{i \mid I}=\hat{a}_{i}-Q_{i I} Q_{I}^{-1}\left(\hat{a}_{I}-z_{I}\right)$

The estimator $\hat{a}_{i \mid I}$ is referred to as the conditional leastsquares ambiguity estimator. It is conditioned on fixing the previous ambiguities to the values $z_{j}, j=1, \ldots,(i-$ 1). Note that $\hat{a}_{i \mid I}$ and $\hat{a}_{I}$ are uncorrelated. This is an important property that will be used repeatedly in the following.

The above result can be used to derive a sequential version of the conditional least-squares estimator. For $i=2$, we obtain the scalar version of (6) as

$\hat{a}_{2 \mid 1}=\hat{a}_{2}-\sigma_{21} \sigma_{1}^{-2}\left(\hat{a}_{1}-z_{1}\right)$

in which $\hat{a}_{2 \mid 1}$ is uncorrelated with $\hat{a}_{1}$. For $i=3$, the conditional least-squares estimator $\hat{a}_{3 \mid 2,1}$ follows from fixing the two ambiguities $a_{1}$ and $a_{2}$ to the values $z_{1}$ and $z_{2}$. Note, however, because $\hat{a}_{3 \mid 2,1}$ is invariant to any regular transformation of $\hat{a}_{1}, \hat{a}_{2}$, that we may as well fix $\hat{a}_{1}$ and $\hat{a}_{2 \mid 1}$ to the values $z_{1}$ and $z_{2}$. This has the advantage that matrix $Q_{I}$ of (6) becomes diagonal. As a result, we obtain

$\hat{a}_{3 \mid 2,1}=\hat{a}_{3}-\sigma_{3,1} \sigma_{1}^{-2}\left(\hat{a}_{1}-z_{1}\right)-\sigma_{3,2 \mid 1} \sigma_{2 \mid 1}^{-2}\left(\hat{a}_{2 \mid 1}-z_{2}\right)$

in which $\hat{a}_{3 \mid 2,1}$ is uncorrelated with both $\hat{a}_{1}$ and $\hat{a}_{2 \mid 1}$. It will be clear that we may continue in this way to obtain the corresponding expressions for the next and following ambiguities as well. The result is summarized as follows. Sequential conditional least-squares The conditional least-squares estimator $\hat{a}_{i \mid I}$ can be computed sequentially as

$\hat{a}_{i \mid I}=\hat{a}_{i}-\sum_{j=1}^{i-1} \sigma_{i, j \mid J} \sigma_{j \mid J}^{-2}\left(\hat{a}_{j \mid J}-z_{j}\right), \quad i=1, \ldots, n$ 
where $\sigma_{i, j \mid J}$ denotes the covariance between $\hat{a}_{i}$ and $\hat{a}_{j \mid J}$, and $\sigma_{j \mid J}^{2}$ is the variance of $\hat{a}_{j \mid J}$. For $i=1, \hat{a}_{i \mid I}$ is set equal to $\hat{a}_{1}$.

We are now in a position to describe the integer bootstrapping principle. In order to compute the sequential conditional least-squares solutions, one needs to specify the $z_{j}$ on which the conditioning takes place. In case of bootstrapping, $z_{j}$, for $j=1, \ldots, n$, is chosen as the nearest integer of $\hat{a}_{j \mid J}$. Hence, for $\hat{a}_{i \mid I}$ the conditioning takes place on the nearest integers of all previous $i-1$ conditional estimates. The $i$ th component of the bootstrapped solution itself is then given as the nearest integer of $\hat{a}_{i \mid I}$. We thus have the following definition.

Definition [Integer bootstrapping] Let $\hat{a}=\left(\hat{a}_{1}, \ldots, \hat{a}_{n}\right)^{\mathrm{T}}$ $\in R^{n}$ be the ambiguity float solution and let $\check{a}_{\mathrm{B}}$ $=\left(\check{a}_{\mathrm{B}, 1}, \ldots, \breve{a}_{\mathrm{B}, \mathrm{n}}\right)^{\mathrm{T}} \in Z^{n}$ denote the corresponding integer bootstrapped solution. The entries of the bootstrapped ambiguity estimator are then defined as

$$
\begin{aligned}
\check{a}_{\mathrm{B}, 1} & =\left[\hat{a}_{1}\right] \\
\check{a}_{\mathrm{B}, 2} & =\left[\hat{a}_{2 \mid 1}\right]=\left[\hat{a}_{2}-\sigma_{21} \sigma_{1}^{-2}\left(\hat{a}_{1}-\check{a}_{\mathrm{B}, 1}\right)\right] \\
& \vdots \\
\check{a}_{\mathrm{B}, \mathrm{n}} & =\left[\hat{a}_{n \mid N}\right]=\left[\hat{a}_{n}-\sum_{j=1}^{n-1} \sigma_{n, j \mid J} \sigma_{j \mid J}^{-2}\left(\hat{a}_{j \mid J}-\check{a}_{\mathrm{B}, \mathrm{j}}\right)\right]
\end{aligned}
$$

where '[.]' denotes the operation of rounding to the nearest integer.

As this definition shows, the bootstrapped estimator can be seen as a generalization of the method of 'integer rounding'. If $n$ ambiguities are available, one starts with the first ambiguity $\hat{a}_{1}$ and rounds its value to the nearest integer. Having obtained the integer value of this first ambiguity, the real-valued estimates of all remaining ambiguities are then corrected by virtue of their correlation with the first ambiguity. Then the second, but now corrected, real-valued ambiguity estimate is rounded to its nearest integer. Having obtained the integer value of the second ambiguity, the real-valued estimates of all remaining $n-2$ ambiguities are then again corrected, but now by virtue of their correlation with the second ambiguity. This process is continued until all ambiguities are accommodated. Thus the bootstrapped estimator reduces to 'integer rounding' in the case that correlations are absent, i.e. in case the ambiguity variance matrix is diagonal.

Note that the bootstrapped estimator is not unique. Changing the order in which the ambiguities appear in vector $\hat{a}$ will already produce a different bootstrapped estimator. Although the principle of bootstrapping remains the same, every choice of ambiguity parametrization has its own bootstrapped estimator.

\subsection{The class of sequential integer estimators}

The earlier bootstrapped estimator is member of a wider class of sequential integer estimators. This class is defined as follows.

Definition [Sequential integer estimation] Let $\hat{a}$ $=\left(\hat{a}_{1}, \ldots, \hat{a}_{n}\right)^{\mathrm{T}} \in R^{n}$ be the ambiguity float solution. Then $\breve{a}=\left(\check{a}_{1}, \ldots, \check{a}_{n}\right)^{\mathrm{T}} \in Z^{n}$ is a sequential integer estimator if $\check{a}_{i}=\left[\hat{a}_{i}+\sum_{j=1}^{i-1} r_{i j}\left(\hat{a}_{j}-\check{a}_{j}\right)\right], i=1, \ldots, n$, or, in vector-matrix form, if

$\check{a}=\left[\hat{a}+\left(R-I_{n}\right)(\hat{a}-\check{a})\right]$

with $R$ a unit lower triangular matrix and where '[.]' denotes componentwise rounding to the nearest integer.

We now show that the bootstrapped estimator $\check{a}_{B}$ is indeed a member of this class. We have the following result.

Theorem 1 Let $\hat{a} \in R^{n}$ be the ambiguity float solution and let the unit lower triangular decomposition of its variance matrix be given as $Q_{\hat{a}}=L D L^{\mathrm{T}}$. The entries of $L$ and $D$ are then given as

$(L)_{i j}=\left\{\begin{array}{cl}0 & \text { for } 1 \leq i<j \leq n \\ 1 & \text { for } i=j \\ \sigma_{i, j \mid j} \sigma_{j \mid J}^{-2} & \text { for } 1 \leq j<i \leq n\end{array}\right.$ and $D=\operatorname{diag}\left(\ldots, \sigma_{j \mid J}^{2}, \ldots\right)$

and the bootstrapped estimator $\check{a}_{\mathrm{B}} \in Z^{n}$ of (10) can be expressed as

$\check{a}_{\mathrm{B}}=\left[\hat{a}+\left(L^{-1}-I_{n}\right)\left(\hat{a}-\check{a}_{\mathrm{B}}\right)\right]$

Proof From (9), it follows that the difference $\left(\hat{a}_{i}-z_{i}\right)$ may be written in terms of the differences $\left(\hat{a}_{j \mid J}-z_{j}\right), j=$ $1, \ldots, i$, as $\left(\hat{a}_{i}-z_{i}\right)=\left(\hat{a}_{i \mid I}-z_{i}\right)+\sum_{j=1}^{i-1} \sigma_{i, j \mid J} \sigma_{j \mid J}^{-2}\left(\hat{a}_{j \mid J}-z_{j}\right)$. When written out in vector-matrix form, this gives

$\left[\begin{array}{c}\hat{a}_{1}-z_{1} \\ \hat{a}_{2}-z_{2} \\ \vdots \\ \hat{a}_{n}-z_{n}\end{array}\right]=\left[\begin{array}{ccc}1 & & \\ l_{21} & 1 & \\ \vdots & \vdots & \ddots \\ l_{n 1} & l_{n 2} \ldots & \ldots\end{array}\right]\left[\begin{array}{c}\hat{a}_{1}-z_{1} \\ \hat{a}_{2 \mid 1}-z_{2} \\ \vdots \\ \hat{a}_{n \mid N}-z_{n}\end{array}\right]$

with $l_{i j}=\sigma_{i, j \mid J} \sigma_{j \mid J}^{-2}$, for $1 \leq j<i \leq n$. Since the sequential conditional least-squares ambiguities are mutually uncorrelated, their variance matrix is diagonal. As a consequence the variance matrix of the $\hat{a}_{i}$ is given a triangular decomposition when the error propagation law is applied to (14). We therefore have the following relation between $\hat{a}=\left(\hat{a}_{1}, \ldots, \hat{a}_{n}\right)^{\mathrm{T}}, \hat{a}_{c}=\left(\hat{a}_{1}, \hat{a}_{2 \mid 1}, \ldots, \hat{a}_{n \mid N}\right)^{\mathrm{T}}$ and the unit lower triangular decomposition of the ambiguity variance matrix: $\hat{a}-z=L\left(\hat{a}_{c}-z\right)$ and $Q_{\hat{a}}=L D L^{\mathrm{T}}$. If we rewrite $\hat{a}-z=L\left(\hat{a}_{c}-z\right)$ as $\hat{a}_{c}=\hat{a}+\left(L^{-1}-I_{n}\right)(\hat{a}-z)$ and use $z=\left[\hat{a}_{c}\right]=\check{a}_{\mathrm{B}}$, the result $\check{a}_{\mathrm{B}}=\left[\hat{a}+\left(L^{-1}-I_{n}\right)\left(\hat{a}-\check{a}_{\mathrm{B}}\right)\right]$ follows. 
With the bootstrapped estimator being a member of the class of sequential integer estimators, one may wonder how its performance compares with the performance of other members from this class. In Sect. 4 , it will be shown that the bootstrapped estimator $\check{a}_{\mathrm{B}}$ is the optimal estimator of this class.

Note that the bootstrapped estimator is determined by the triangular matrix $L$. Hence, the estimator takes only part of the information of the ambiguity variance matrix $Q_{\hat{a}}$ into account. The matrix $D$ of conditional variances does not play a role in the bootstrapped mapping. But as we will see in the next section, matrix $D$ contains all the information for determining the success rate of bootstrapping.

Equation (13) provides an alternative way of computing the bootstrapped estimator, one which is particularly useful when the unit upper triangular decomposition of the inverse of $Q_{\hat{a}}$ is given. Let this decomposition be given as $Q_{\hat{a}}^{-1}=U \Lambda U^{T}$. Then $Q_{\hat{a}}=U^{-T} \Lambda^{-1} U^{-1}=$ $L D L^{T}$. From the uniqueness of the triangular decomposition, it follows that $L^{-1}=U^{T}$ and $D=\Lambda^{-1}$. Thus if the unit upper triangular decomposition of the inverse of the variance matrix is given, one can compute the bootstrapped estimator using $U^{T}$.

\subsection{The probability mass function of the ambiguity bootstrapped estimator}

Let $B$ denote the bootstrapped mapping. Then $B: R^{n} \mapsto$ $Z^{n}$, because the bootstrapped estimator maps the realvalued float ambiguity vector $\hat{a}$ to the integer vector $\check{a}_{\mathrm{B}}$. Since the bootstrapped estimator maps different realvalued ambiguity vectors to the same integer vector, the bootstrapped estimator is a many-to-one map. One can therefore assign a subset $B_{z} \subset R^{n}$ to each integer vector $z \in Z^{n}$ as

$B_{z}=\left\{x \in R^{n} \mid z=B(x)\right\}, \quad z \in Z^{n}$

The subset $B_{z}$ contains all real-valued ambiguity vectors that will be mapped by $B$ to the same integer vector $z \in Z^{n}$. This subset is referred to as the bootstrapped pull-in region of $z$ (Jonkman 1998; Teunissen 1998). It is the region from which all ambiguity float solutions are pulled to the same fixed ambiguity vector $z$.

The bootstrapped pull-in regions are given as

$$
B_{z}=\left\{x \in R^{n}|| c_{i}^{T} L^{-1}(x-z) \mid \leq \frac{1}{2}, i=1, \ldots, n\right\}, \quad \forall z \in Z^{n}
$$

where $L$ is the unit lower triangular matrix of $Q_{\hat{a}}=$ $L D L^{\mathrm{T}}$ and $c_{i}$ denotes the $i$ th canonical unit vector having a 1 as its $i$ th entry and zeros otherwise. To see this, consider the relation $\hat{a}_{c}-z=L^{-1}(\hat{a}-z)$. According to (10), the integer vector $z$ equals the bootstrapped solution when rounding to the nearest integer of each of the components of $\hat{a}_{c}-z$ gives zero, or similarly, when the absolute values of these components are all less than or equal to $1 / 2$. Since this is equivalent to stating that the absolute values of all the components of $L^{-1}(\hat{a}-z)$ are required to be less than or equal to $1 / 2$, the result (16) follows.

The bootstrapped pull-in regions are translated copies of each another $\left(B_{z}=z+B_{0}, \forall z \in Z^{n}\right)$ and cover the whole space without gaps and overlaps $\left(\cup_{z \in Z^{n}} B_{z}=\right.$ $R^{n}$ and $\left.\operatorname{Int} B_{z_{1}} \cap \operatorname{Int} B_{z_{2}}=\emptyset, \forall z_{1}, z_{2} \in Z^{n}, z_{1} \neq z_{2}\right)$.

The bootstrapped pull-in regions can be used to determine the distribution of the bootstrapped estimator. Since $\check{a}_{\mathrm{B}}=z \Longleftrightarrow \hat{a} \in B_{z}$ and $\hat{a} \sim \mathrm{N}\left(a, Q_{\hat{a}}\right)$, the probability mass function (PMF) of $\check{a}_{\mathrm{B}}$ is given as

$$
\begin{aligned}
P\left(\check{a}_{\mathrm{B}}=z\right)= & \int_{B_{z}}(2 \pi)^{-\frac{n}{2}} \sqrt{\operatorname{det} Q_{\hat{a}}^{-1}} \\
& \exp \left\{-\frac{1}{2}(x-a)^{T} Q_{\hat{a}}^{-1}(x-a)\right\} \mathrm{d} x, \quad \forall z \in Z^{n}
\end{aligned}
$$

It is the integral of the multivariate normal distribution over the bootstrapped pull-in region $B_{z}$. As the following theorem shows, the multivariate integral can be expressed as a product of $n$ univariate integrals.

Theorem 2 Let $\hat{a}$ be distributed as $\mathrm{N}\left(a, Q_{\hat{a}}\right), a \in Z^{n}$, and let $\breve{a}_{\mathrm{B}}$ be the corresponding integer bootstrapped estimator. Then

$$
\begin{aligned}
P\left(\check{a}_{\mathrm{B}}=z\right)=\prod_{i=1}^{n}[ & \Phi\left(\frac{1-2 l_{i}^{T}(a-z)}{2 \sigma_{\hat{a}_{i \mid I}}}\right) \\
& \left.+\Phi\left(\frac{1+2 l_{i}^{T}(a-z)}{2 \sigma_{\hat{a}_{i \mid I}}}\right)-1\right], \quad \forall z \in Z^{n}
\end{aligned}
$$

with

$\Phi(x)=\int_{-\infty}^{x} \frac{1}{\sqrt{2 \pi}} \exp \left\{-\frac{1}{2} v^{2}\right\} \mathrm{d} v$

and where $l_{i}$ is the ith column vector of the unit upper triangular matrix $L^{-T}$ and $\sigma_{\hat{a}_{i \mid I}^{2}}^{2}$ is the variance of the ith least- squares ambiguity obtained through a conditioning on the previous $I=\{1, \ldots,(i-1)\}$ ambiguities.

The proof is given in Teunissen (2001). Note that the bootstrapped PMF is symmetric about the mean of $\hat{a}$. This implies that the bootstrapped estimator $\check{a}_{\mathrm{B}}$ is an unbiased estimator of $a \in Z^{n}$. Also observe that the shape of the bootstrapped PMF is completely governed by the ambiguity variance matrix $Q_{\hat{a}}$. The PMF follows once the triangular factor $L$ and the diagonal matrix $D$ 
of the decomposition $Q_{\hat{a}}=L D L^{\mathrm{T}}$ are given. Finally note that the PMF reaches its maximum at its point of symmetry. Thus $\max _{z} P\left(\check{a}_{\mathrm{B}}=z\right)=P(\check{a}=a)$. This is a reassuring result, since it implies that the bootstrap probability of correct integer estimation is largest of all probability masses. This probability will be referred to as the bootstrapped success rate.

\section{The effect of improving the ambiguity precision}

The goal of ambiguity resolution is to estimate the unknown integer ambiguity vector $a$. We know that the integer bootstrapped estimator is unbiased, i.e. $E\left(\breve{a}_{\mathrm{B}}\right)=$ $a$. This is a nice result, since it implies that one can expect the outcome of $\check{a}_{\mathrm{B}}$ to be correct on the average. In order to judge the performance of the integer estimator, however, the property of unbiasedness is too weak a property to rely on. What we need is the frequency with which one can expect to obtain correct results. This frequency is provided by the probability of correct integer estimation, the success rate $P\left(\check{a}_{\mathrm{B}}=a\right)$. The bootstrapped success rate follows from setting $z=a$ in (18), as

$P\left(\check{a}_{\mathrm{B}}=a\right)=\prod_{i=1}^{n}\left[2 \Phi\left(\frac{1}{2 \sigma_{\hat{a}_{i \mid I}}}\right)-1\right]$

Note that it is completely driven by the sequential conditional variances $\sigma_{\hat{a}_{i \mid} \mid}^{2}$, and thus by the entries of the diagonal matrix $D$ in the triangular decomposition $Q_{\hat{a}}=$ $L D L^{\mathrm{T}}$

As was mentioned earlier, the outcome of bootstrapping depends on the chosen ambiguity parametrization. Bootstrapping of DD ambiguities, for instance, will produce an integer solution that generally differs from the integer solution obtained from bootstrapping of reparametrized ambiguities. Since this dependency also holds true for the bootstrapped PMF, one has some important degrees of freedom left for improving (19).

In order to improve the bootstrapped success rate, one should work with decorrelated ambiguities instead of with the original ambiguities. The method of bootstrapping performs relatively poorly, for instance, when applied to the DD ambiguities. This is due to the usually high correlation between the DD ambiguities. Bootstrapping should therefore be used in combination with a volume preserving decorrelating $Z$-transformation. Such a transformation reduces the sequential conditional variances and therefore enlarges the bootstrapped success rate. Thus if $\hat{a}$ is the DD float solution, a larger success rate is possible if bootstrapping is applied to $\hat{z}=Z \hat{a}$. In case of multi-frequency GNSS, an example of such a $Z$-transformation is provided by the transformation to widelane ambiguities. This has been demon- strated analytically in Teunissen (1997). However, one can even do better than this by using the decorrelating $Z$-transformation of the LAMBDA method. This transformation decorrelates the ambiguities further and thereby achieves a further reduction of the values of the sequential conditional variances. For more information on the LAMBDA method, the reader is referred to Teunissen $(1993,1995)$ and de Jonge and Tiberius (1996a) or to the textbooks Hofmann-Wellenhof et al. (1997), Strang and Borre (1997), Teunissen and Kleusberg (1998), Misra and Enge (2001). Practical results obtained with it and suggested improvements, can be found, for example, in Boon and Ambrosius (1997), Boon et al. (1997), Chang et al. (2005), Cox and Brading (1999), Dai et al. (2005), de Jonge and Tiberius (1996b), de Jonge et al. (1996), Han (1995), Jonkman (1998), Moenikes et al. (2005), Peng et al. (1999), Svendsen (2005), Tiberius and de Jonge (1995), Tiberius et al. (1997).

The above method of improving the success rate makes use of the lack of invariance of the bootstrapped estimator for ambiguity reparametrizations. It is not based on a change of the strength of the underlying model. It seems reasonable however to ask of an integer estimator that it has the property that its success rate increases when the precision of its input gets better. This property is yet to be proven for the bootstrapped estimator.

Before stating our result, we first specify what we mean by 'better precision'. Let $Q_{1}$ and $Q_{2}$ be the variance matrices of two float solutions $\hat{a}^{Q_{1}}$ and $\hat{a}^{Q_{2}}$. The precision of $\hat{a}^{Q_{1}}$ is then said to be better than the precision of $\hat{a}^{Q_{2}}$, when the variance of every linear function of $\hat{a}^{Q_{1}}$ is smaller than the variance of the same function of $\hat{a}^{Q_{2}}$. Thus $f^{\mathrm{T}} Q_{1} f<f^{\mathrm{T}} Q_{2} f$ must hold for every $f \in R^{n} \backslash\{0\}$. This is equivalent to stating that $Q_{2}>Q_{1}$, or that matrix $Q_{2}-Q_{1}$ is positive definite. We have the following result.

Theorem 3 Let $\hat{a}^{Q} \sim N(a, Q)$, with $a \in Z^{n}$, and let $\check{a}_{\mathrm{B}}^{Q}$ be the corresponding integer bootstrapped estimator of a. Then

$P\left(\check{a}_{\mathrm{B}}^{Q_{1}}=a\right)>P\left(\check{a}_{\mathrm{B}}^{Q_{2}}=a\right) \quad$ if $Q_{2}>Q_{1}$

Proof Let the triangular decompositions of $Q_{1}$ and $Q_{2}$ be given as $Q_{1}=L_{1} D_{1} L_{1}^{\mathrm{T}}$ and $Q_{2}=L_{2} D_{2} L_{2}^{\mathrm{T}}$, respectively. Furthermore, define $f=L_{2}^{-\mathrm{T}} g$, the unit lower triangular matrix $L=L_{2}^{-1} L_{1}$ and the canonical unit vector $c_{i}=(\ldots, 0,1,0, \ldots)^{\mathrm{T}}$ having a one as its $i$ th entry and zeros otherwise. Then 


$$
\begin{aligned}
Q_{2} & >Q_{1} \\
& \Leftrightarrow f^{\mathrm{T}} Q_{2} f>f^{\mathrm{T}} Q_{1} f, \quad \forall f \in R^{n} \backslash\{0\} \\
& \Leftrightarrow f^{\mathrm{T}} L_{2} D_{2} L_{2}^{\mathrm{T}} f>f^{\mathrm{T}} L_{1} D_{1} L_{1}^{\mathrm{T}} f, \quad \forall f \in R^{n} \backslash\{0\} \\
& \Leftrightarrow g^{\mathrm{T}} D_{2} g>g^{\mathrm{T}} L D_{1} L^{\mathrm{T}} g, \quad \forall g \in R^{n} \backslash\{0\} \\
& \Rightarrow c_{i}^{\mathrm{T}} D_{2} c_{i}>c_{i}^{\mathrm{T}} L D_{1} L^{\mathrm{T}} c_{i}, \quad i=1, \ldots, n \\
& \Leftrightarrow\left(D_{2}\right)_{i i}>\left(D_{1}\right)_{i i}+\sum_{j=1}^{i-1}(L)_{i j}^{2}\left(D_{1}\right)_{j j}, \quad i=1, \ldots, n \\
& \Leftrightarrow\left(D_{2}\right)_{i i}>\left(D_{1}\right)_{i i}, \quad i=1, \ldots, n
\end{aligned}
$$

This shows that $Q_{2}>Q_{1}$ implies that the sequential conditional variances of $Q_{2}$ are always strictly larger than their counterparts of $Q_{1}$. This proves (20). Note that the converse is not true. That is, $P\left(\check{a}_{\mathrm{B}}^{Q_{1}}=a\right)>$ $P\left(\check{a}_{\mathrm{B}}^{Q_{2}}=a\right)$ does not imply that $Q_{2}>Q_{1}$.

The above result states that the bootstrapped success rate always gets larger when the precision of the float solution improves. Thus every precision improvement that one can realize in the underlying model (e.g. by including more data or more precise data) will directly benefit the bootstrapped ambiguity resolution. The above result also implies that in case the inverse variance matrix of the observations is used as weight matrix, the least-squares method is the best method for computing the float solution. Such a least-squares estimator is namely known to be a best linear unbiased estimator. It is the estimator which has the best precision of all linear unbiased estimators.

\section{The effect of using an approximate ambiguity variance matrix}

Apart from knowing the relation between the success rate and the actual ambiguity precision, it is also of importance to know the relation between the success rate and a presumed ambiguity precision. In other words, what happens to the success rate if the computed bootstrapped estimator is based on a too optimistic description of the ambiguity precision or on a too pessimistic description of the ambiguity precision? In either case, one would want the success rate not to increase. This property is yet to be proven for the bootstrapped estimator. The proof is given by the following theorem.

Theorem 4 Let $\hat{a} \sim N(a, Q)$, with $a \in Z^{n}$, and let $\check{a}_{\mathrm{B}}^{Q}$ be the corresponding integer bootstrapped estimator of a. Furthermore let $\breve{a}_{\mathrm{B}}^{\Sigma}$ be the integer bootstrapped estimator constructed on the basis of the positive definite matrix $\Sigma$ instead of $Q$. Then
$P\left(\check{a}_{\mathrm{B}}^{\Sigma}=a\right) \leq P\left(\check{a}_{\mathrm{B}}^{Q}=a\right)$

with strict inequality if the unit triangular factors of $\Sigma$ and $Q$ differ.

Proof Let the triangular decompositions of $\Sigma$ and $Q$ be given as $\Sigma=L_{\Sigma} D_{\Sigma} L_{\Sigma}^{\mathrm{T}}$ and $Q=L_{Q} D_{Q} L_{Q}^{\mathrm{T}}$, respectively. Then

$$
\begin{aligned}
& P\left(\check{a}_{\mathrm{B}}^{\Sigma}=a\right)=\frac{1}{(2 \pi)^{n / 2}} \int_{B_{\Sigma, a}} \frac{1}{\left(\operatorname{det} D_{Q}\right)^{1 / 2}} \\
& \quad \times \exp \left\{-\frac{1}{2}(x-a)^{\mathrm{T}}\left(L_{Q} D_{Q} L_{Q}^{\mathrm{T}}\right)^{-1}(x-a)\right\} \mathrm{d} x \\
& P\left(\check{a}_{\mathrm{B}}^{Q}=a\right)=\frac{1}{(2 \pi)^{n / 2}} \int_{B_{Q, a}} \frac{1}{\left(\operatorname{det} D_{Q}\right)^{1 / 2}} \\
& \quad \times \exp \left\{-\frac{1}{2}(x-a)^{\mathrm{T}}\left(L_{Q} D_{Q} L_{Q}^{\mathrm{T}}\right)^{-1}(x-a)\right\} \mathrm{d} x
\end{aligned}
$$

with pull-in regions

$$
\begin{aligned}
& B_{\Sigma, a}=\left\{x \in R^{n}|| c_{i}^{\mathrm{T}} L_{\Sigma}^{-1}(x-a) \mid \leq \frac{1}{2}, i=1, \ldots, n\right\} \\
& B_{Q, a}=\left\{x \in R^{n}|| c_{i}^{\mathrm{T}} L_{Q}^{-1}(x-a) \mid \leq \frac{1}{2}, i=1, \ldots, n\right\}
\end{aligned}
$$

Note that the above two integrals only differ in their region of integration. Using the change of variables formula for integrals, we now apply the transformation $T$ : $x=L_{Q} y+a$ to both integrals. This gives,

$$
\begin{aligned}
& P\left(\check{a}_{\mathrm{B}}^{\Sigma}=a\right)=\frac{1}{(2 \pi)^{n / 2}} \int_{T^{-1}\left(B_{\Sigma, a}\right)} \frac{1}{\left(\operatorname{det} D_{Q}\right)^{1 / 2}} \\
& \quad \times \exp \left\{-\frac{1}{2} y^{\mathrm{T}} D_{Q}^{-1} y\right\} \mathrm{d} y \\
& P\left(\check{a}_{\mathrm{B}}^{Q}=a\right)=\frac{1}{(2 \pi)^{n / 2}} \int_{T^{-1}\left(B_{Q, a}\right)} \frac{1}{\left(\operatorname{det} D_{Q}\right)^{1 / 2}} \\
& \quad \times \exp \left\{-\frac{1}{2} y^{\mathrm{T}} D_{Q}^{-1} y\right\} \mathrm{d} y
\end{aligned}
$$

where

$$
\begin{aligned}
& T^{-1}\left(B_{\Sigma, a}\right)=\left\{y \in R^{n}|| c_{i}^{\mathrm{T}} L y \mid \leq \frac{1}{2}, i=1, \ldots, n\right\} \\
& T^{-1}\left(B_{Q, a}\right)=\left\{y \in R^{n}|| c_{i}^{\mathrm{T}} y \mid \leq \frac{1}{2}, i=1, \ldots, n\right\}
\end{aligned}
$$

with the unit lower triangular matrix $L=L_{\Sigma}^{-1} L_{Q}$. Note that the two integrals have identical outcomes if $L=I_{n}$, that is, if $L_{\Sigma}=L_{Q}$. In all other cases, their outcomes will differ. For these cases we now show that $P\left(\breve{a}_{\mathrm{B}}^{\Sigma}=\right.$ $a)<P\left(\check{a}_{\mathrm{B}}^{Q}=a\right)$. With $D_{Q}=\operatorname{diag}\left(\sigma_{1}^{2}, \sigma_{2 \mid 1}^{2}, \ldots, \sigma_{n \mid N}^{2}\right)$ and $(L)_{i j}=l_{i j}$, we may write the multivariate integral of $P\left(\check{a}_{\mathrm{B}}^{\Sigma}=a\right)$ as a string of nested univariate integrals, 
$P\left(\breve{a}_{\mathrm{B}}^{\Sigma}=a\right)=\int_{i_{1}} \frac{\exp \left\{-\frac{1}{2} \frac{y_{1}^{2}}{\sigma_{1}^{2}}\right\}}{\sigma_{1} \sqrt{2 \pi}}\left(\ldots\left(\int_{i_{n-1}} \frac{\exp \left\{-\frac{1}{2} \frac{y_{n-1}^{2}}{\sigma_{n-1 \mid N-1}^{2}}\right\}}{\sigma_{n-1 \mid N-1} \sqrt{2 \pi}}\left(\int_{i_{n}} \frac{\exp \left\{-\frac{1}{2} \frac{y_{n}^{2}}{\sigma_{n \mid N}^{2}}\right\}}{\sigma_{n \mid N} \sqrt{2 \pi}} \mathrm{d} y_{n}\right) \mathrm{d} y_{n-1}\right) \ldots\right) \mathrm{d} y_{1}$

with the $n$ intervals

$i_{1}:\left|y_{1}\right| \leq 1 / 2$

$i_{2}:\left|l_{21} y_{1}+y_{2}\right| \leq 1 / 2$

$\vdots \vdots$

$i_{n}:\left|l_{n 1} y_{1}+l_{n 2} y_{2}+\cdots+l_{n, n-1} y_{n-1}+y_{n}\right| \leq 1 / 2$

For the innermost integral we have the inequality

$$
\begin{aligned}
& \int_{i_{n}} \frac{\exp \left\{-\frac{1}{2} \frac{y_{n}^{2}}{\left.\sigma_{n \mid N}^{2}\right\}}\right.}{\sigma_{n \mid N} \sqrt{2 \pi}} \mathrm{d} y_{n} \\
& \quad<\int_{\left|y_{n}\right| \leq 1 / 2} \frac{\exp \left\{-\frac{1}{2} \frac{y_{n}^{2}}{\sigma_{n \mid N}^{2}}\right\}}{\sigma_{n \mid N} \sqrt{2 \pi}} \mathrm{d} y_{n}=\left(2 \Phi\left(\frac{1}{2 \sigma_{n \mid N}}\right)-1\right)
\end{aligned}
$$

since the interval $\left|y_{n}\right| \leq 1 / 2$ is symmetric with respect to the origin, whereas the interval $i_{n}$, which has the same length as $\left|y_{n}\right| \leq 1 / 2$, is not symmetric with respect to the origin. Proceeding in this fashion from the innermost integral to the outer integral, we obtain the inequality

$$
P\left(\check{a}_{\mathrm{B}}^{\Sigma}=a\right)<\prod_{i=1}^{n}\left(2 \Phi\left(\frac{1}{2 \sigma_{i \mid I}}\right)-1\right)=P\left(\check{a}_{\mathrm{B}}^{Q}=a\right)
$$

This theorem shows that, with one exception, the use of an improper ambiguity variance matrix (too optimistic or too pessimistic) will always result in a smaller bootstrapped success rate. The exception occurs when the variance matrix $Q$ and its approximation $\Sigma$ have the same triangular factor. This case, however, is not likely to occur in practice. The conclusion reads therefore that also in case of integer bootstrapping it directly pays off to improve upon the approximation of the underlying mathematical model. For GNSS, the functional model (observation equations) is sufficiently known and well documented. The same cannot yet be said however of the precision description of the GNSS data. Of course, a systematic study of the stochastic model is far from trivial. Not only do the noise characteristics depend on the mechanization of the measurement process and therefore on the make and type of the receiver used, but the random residual terms such as environmental effects, will also have their influence. Fortunately the interest in the topic of improved stochastic modelling is gaining ground in GNSS research and will have a positive effect on the bootstrapped success rate.

It was shown that the bootstrapped estimator is a member of the class of sequential integer estimators. With the above result, we have as a direct by-product that the bootstrapped estimator is the optimal estimator within this restricted class.

Corollary Let $\hat{a} \sim N(a, Q)$, with $a \in Z^{n}$, and let $\check{a}_{\mathrm{B}}^{Q}$ be the corresponding integer bootstrapped estimator of a. Then

$P\left(\check{a}_{\mathrm{B}}^{Q}=a\right) \geq P(\check{a}=a)$

for any sequential integer estimator $\check{a}=\left[\hat{a}+\left(R-I_{n}\right)(\hat{a}-\right.$ $\breve{a})$ ], where $R$ is a unit lower triangular matrix.

Note, when $R$ is chosen as $R=I_{n}$, that the sequential integer estimator reduces to $\check{a}=[\hat{a}]$, i.e. the integer estimator based on componentwise rounding. Hence, as another by-product, we have that $P\left(\check{a}_{\mathrm{B}}^{Q}=a\right) \geq P([\hat{a}]=$ a). Thus the success rate of componentwise rounding will never be larger than the bootstrapped success rate.

\section{Concluding remarks}

Integer ambiguity bootstrapping is, because of its ease of computation, a popular method for resolving the integer GNSS carrier phase ambiguities. The method is, however, suboptimal, since it only takes part of the information from the ambiguity variance matrix into account. It is therefore of importance to know how the success rate (i.e. the probability of correct integer estimation) of integer bootstrapping relates to the precision of the 'float' ambiguities. The following two cases were considered in this contribution: (1) the effect of improving the ambiguity precision, and (2) the effect of using an incorrect, or approximate, ambiguity variance matrix.

It was shown that the bootstrapped success rate always gets larger, if the precision of the 'float' ambiguities improves. This is a reassuring result, since it implies that integer bootstrapping will always benefit from a strengthening of the underlying GNSS model. The result also implies, since properly weighted least-squares estimators have the property of minimum variance (BLUE), 
that one should use the principle of least-squares for computing the 'float' solution.

We also investigated what happens to the success rate of integer bootstrapping, if an incorrect ambiguity variance matrix is used (either too optimistic or too pessimistic) for computing the bootstrapped solution. It was shown that the success rate of integer bootstrapping based on an incorrect ambiguity variance matrix is always less than or equal to the success rate of integer bootstrapping based on the correct ambiguity variance matrix. There is a strict inequality between the two success rates, if the unit triangular factor of the incorrect ambiguity variance matrix differs from the unit triangular factor of the correct ambiguity variance matrix.

The ease with which integer bootstrapping can be computed stems from its sequential character. That is, in contrast with, for instance, integer least-squares, no integer search needs to be performed for integer bootstrapping. We defined the class of integer sequential estimators and showed that integer bootstrapping is a member of this class. Although integer bootstrapping is suboptimal in the class of integer estimators, we have shown that it is optimal within the more restricted class of integer sequential estimators. Hence, just like integer least-squares has the largest possible success rate of all integer estimators, integer bootstrapping has the largest possible success rate of all integer sequential estimators.

\section{References}

Boon F, Ambrosius B (1997) Results of real-time applications of the LAMBDA method in GPS based aircraft landings. In: Proceedings KIS97, pp. 339-345

Boon F, de Jonge PJ, Tiberius CCJM (1997) Precise aircraft positioning by fast ambiguity resolution using improved troposphere modelling. In: Proceedings ION GPS-97, vol 2, pp 1877-1884

Chang XW, Yang X, Zhou T (2005) MLAMBDA: a modified LAMBDA method for integer ambiguity determination. In: Proceedings ION GNSS2005, Long Beach, CA, USA

Cox DB, Brading JDW (1999) Integration of LAMBDA ambiguity resolution with Kalman filter for relative navigation of spacecraft. In: Proceedings ION NTM 99, pp 739-745

Dai L, Nagarajan N, Hu G, Ling K (2005) Real-time attitude determination for micro satellites by LAMBDA method combined with Kalman filtering. In: AIAA proceedings 22nd ICSSC, Monterey, CA, USA

de Jonge PJ, Tiberius CCJM (1996a) The LAMBDA method for integer ambiguity estimation: implementation aspects. Publications of the Delft Computing Centre, LGR-Series No. 12

de Jonge PJ, Tiberius CCJM (1996b) Integer estimation with the LAMBDA method. In: Beutler G et al. (eds) Proceedings IAG Symposium No. 115, GPS trends in terrestrial, airborne and spaceborne applications. Springer, Berlin Heidelberg New York, pp 280-284 de Jonge PJ, Tiberius CCJM, Teunissen PJG (1996) Computational aspects of the LAMBDA method for GPS ambiguity resolution. In: Proceedings ION GPS-96, pp 935944

Han S (1995) Ambiguity resolution techniques using integer leastsquares estimation for rapid static or kinematic positioning. Symposium Satellite Navigation Technology: 1995 and beyond, Brisbane, $10 \mathrm{pp}$

Hofmann-Wellenhof B, Lichtenegger H, Collins J (1997) Global positioning system: theory and practice, 4th edn. Springer, Berlin Heidelberg New York

Jonkman NF (1998) Integer ambiguity estimation without the receiver-satellite geometry. Publications of the Delft Geodetic computing centre, LGR-Series, No. 18

Leick A (1995) GPS satellite surveying, 2nd edn. Wiley, New York

Misra P, Enge P (2001) Global positioning system: signals, measurements, and performance. Ganga-Jamuna Press

Moenikes R, Wendel J, Trommer GF (2005) A modified LAMBDA method for ambiguity resolution in the presence of position domain constraints. In: Proceedings ION GNSS2005, Long Beach, CA, USA

Parkinson B, Spilker JJ (eds) (1996) GPS: theory and applications. vols 1 and 2, AIAA, Washington DC

Peng HM, Chang FR, Wang LS (1999) Attitude determination using GPS carrier phase and compass data. In: Proceedings ION NTM 99, pp 727-732

Strang G, Borre K (1997) Linear algebra, geodesy, and GPS. Wellesley-Cambridge Press

Svendsen JGG (2005) Some properties of decorrelation techniques in the ambiguity space. GPS Solutions. DOI 10.1007/ s10291-005-0004-6

Teunissen PJG (1993) Least-squares estimation of the integer GPS ambiguities. Invited lecture, Section IV theory and methodology, IAG general meeting, Beijing, China, August 1993. Also in: LGR Series, No. 6, Delft Geodetic computing centre

Teunissen PJG (1995) The least-squares ambiguity decorrelation adjustment: a method for fast GPS integer ambiguity estimation. J Geodesy 70:65-82

Teunissen PJG (1997) On the GPS widelane and its decorrelating property. J Geodesy 71:577-587

Teunissen PJG (1998) On the integer normal distribution of the GPS ambiguities. Artif Satellites 33(2):49-64

Teunissen PJG (1999a) The probability distribution of the GPS baseline for a class of integer ambiguity estimators. J Geodesy 73:275-284

Teunissen PJG (1999b) An optimality property of the integer least-squares estimator. J Geodesy 73:587-593

Teunissen PJG (2000) Adjustment theory. Delft University Press, Delft, The Netherlands

Teunissen PJG (2001) The probability distribution of the ambiguity bootstrapped GNSS baseline. J Geodesy 75:267-275

Teunissen PJG, Kleusberg A (eds) (1998) GPS for geodesy. 2nd enlarged edn. Springer, Berlin Heidelberg New York

Tiberius CCJM, de Jonge PJ (1995) Fast positioning using the LAMBDA method. In: Proceedings DSNS-95, paper 30, 8 pp

Tiberius CCJM, Teunissen PJG, de Jonge PJ (1997) Kinematic GPS: performance and quality control. In: Proceedings KIS97, pp 289-299 